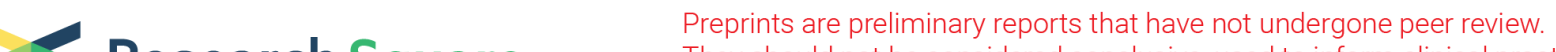 Research Square They should not be considered conclusive, used to inform clinical practice, or referenced by the media as validated information.
}

\section{Comparison of Effects of Guidewire-Assisted and Direct Arterial Puncture Techniques on Success Rate of Radial Artery Cannulation by Inexperienced Trainees}

\section{Worapot Apinyachon}

Department of Anesthesiology, Faculty of Medicine, Ramathibodi Hospital, Mahidol University Natthaporn Boonyakad

Department of Anesthesiology, Faculty of Medicine, Ramathibodi Hospital, Mahidol University Inthuon Sangasilpa

Department of Anesthesiology, Faculty of Medicine, Ramathibodi Hospital, Mahidol University Phisut Lavanrattanakul ( $\square$ kathualavan@gmail.com )

Department of Anesthesiology, Faculty of Medicine, Ramathibodi Hospital, Mahidol University

\section{Research Article}

Keywords: Blood Pressure, Catheterization Peripheral / methods, Catheterization, Peripheral / adverse effects, Internship and Residency, Radial artery, Training Support

Posted Date: August 17th, 2021

DOI: https://doi.org/10.21203/rs.3.rs-601570/v1

License: (c) (i) This work is licensed under a Creative Commons Attribution 4.0 International License. Read Full License 


\section{Abstract}

Background: The radial artery is superficially palpable and easily accessible, making it the preferred site for arterial cannulation. Various placement techniques are available for radial artery catheter insertion. However, catheter insertion is sometimes difficult and requires multiple attempts, especially by inexperienced trainees, leading to technical failure and insertion-related complications. Guidewireassisted cannulation is a simple and effective technique, but this maneuver requires practice. The main objective of this study was to compare the effects of a direct arterial puncture and a guidewire-assisted cannulation technique on the success rate of radial artery cannulation by inexperienced trainees.

Methods: This study was a prospective, single-center, single-arm clinical trial with a historical control. Sixty patients with an American Society of Anesthesiologists physical status of I to III underwent elective surgery requiring radial artery cannulation. In the guidewire-assisted cannulation group (GW group), thirty radial artery cannulations were performed by inexperienced surgical residents using a guidewire-assisted cannulation technique. In the direct arterial puncture group (DP group), 30 patients underwent radial artery cannulation with a direct arterial puncture technique by inexperienced anesthesiology residents. The primary outcome was the success rate.

Results: There were no differences in the baseline blood pressure or comorbidities between the two groups. Overall, the success rate of radial artery cannulation in the GW and DP groups were $90 \%$ and $50 \%$, respectively $(P=0.001)$. The success rate of first-attempt cannulation in the GW and DP group were $66.7 \%$ and $26.7 \%$, respectively $(P=0.002)$. The total procedural performance time was significantly shorter in the GW than DP group.

Conclusion: The guidewire-assisted cannulation technique achieved a higher success rate than the direct arterial puncture technique when performed by inexperienced trainees.

\section{Trial registration}

The trial was registered on Thai Clinical Trials Registry (TCTR20200601004). Date of registration was January 19, 2020. (Retrospectively registered) http://www.thaiclinicaltrials.org/page_user/\#

\section{Background}

Invasive blood pressure monitoring is commonly used in patients requiring continuous blood pressure measurement or frequent blood sampling.[1] The radial artery is the most commonly used artery for this purpose because of its superficial location and fewer complications compared with other sites.[2] Catheter placement can be performed using various techniques, including direct arterial puncture or palpation as well as guidewire-assisted or ultrasound-guided arterial line placement.[3] In training programs, radial artery cannulation is a compulsory procedure for anesthesiology residents. Inevitably, the risks and practice requirements of various procedures are topics of concern in any academic training center. 
We mainly use a direct arterial puncture technique for radial artery catheter insertion at our institute. However, insertion of the radial artery catheter is sometimes difficult and requires multiple attempts, especially for inexperienced trainees; this may lead to insertion failure and insertion-related complications. Unsurprisingly, more experienced trainees have higher cannulation success rates. However, the development of training programs for practicing radial artery cannulation, such as preprocedural simulator training or video demonstrations, can significantly improve the success rate for inexperienced trainees.[4] The most fundamental technique for radial artery cannulation is direct arterial puncture, which is performed without any assisting devices and has a learning curve. Therefore, its failure rate remains high among inexperienced practitioners and even some skilled anesthesiologists. $[4,5]$ The use of a guidewire or the Seldinger technique to aid radial artery cannulation is a reliable method that can achieve a high success rate.[6, 7] However, training and practice are required for practitioners who are unfamiliar with the technique or who have less experience.[8]

The main objective of this study was to compare the effects of direct arterial puncture, which is our standard technique, and a guidewire-assisted cannulation technique on the success rate of radial artery cannulation by inexperienced trainees. We hypothesised that guidewire-assisted cannulation can improve the success rate of cannulation.

\section{Methods}

This study was a prospective, single-center, single-arm clinical trial with a historical control. This study was approved by institutional ethics committee in Mahidol University (046125) and was registered on Thai Clinical Trials Registry (TCTR20200601004). All patients signed a written consent form before their participation in the study that was conducted from February 2019 to September 2019 at Ramathibodi Hospital, Mahidol University. The reporting in this manuscript followed the recommendations in the STROBE guideline.

All patients underwent elective hepatobiliary and pancreatic surgery under general anaesthesia, had an American Society of Anesthesiologists (ASA) physical status of I to III, were older than 18 years, and required radial artery cannulation for intraoperative monitoring. We excluded patients who required insertion of an arterial line before induction of anaesthesia, had previously undergone radial artery cannulation during the same hospital admission, had unstable vital signs, or had peripheral vascular disease. The patients were assigned into two groups; direct arterial puncture group (DP group) and guidewire-assisted cannulation group (GW group). The study was separated to two periods. The DP group was conducted when our new first-year anesthesiology residency trainees started working at the operating room after orientation program. The GW group was conducted in surgical residency trainees who rotated into division of hepatobiliary and pancreatic surgery.

In the direct arterial puncture group, the cannulation procedures were performed by first-year anesthesiology residents who had performed either zero or one radial artery cannulation. They had attended simulator training of radial artery cannulation by direct arterial puncture on a manikin-based 
training and watched a video demonstrating this cannulation technique on the orientation day of our training program. In the guidewire-assisted cannulation group, radial artery cannulation was performed by inexperienced surgical residents who had only performed arterial blood gas sampling and had never performed radial artery cannulation. The study assigned only one anesthesiologist who did not involve with the patient care processes to show the video demonstrating steps of guidewire-assisted cannulation technique to surgical residents on the morning of procedure. After watching video that was approximately seven minutes in length, the surgical residents were able to ask any question before the procedure. The nurse anesthetists prepared radial artery cannulation equipment for our trainees.

Both techniques started with the patient's arm secured on an arm board, the wrist extended, and the fingers fixed. In the DP group, after cleaning the cannulation site with antiseptic solution, the direct arterial puncture began with manual palpation to identify the site of maximal pulsation. After the point of puncture had been identified, a 20-gauge fluorinated ethylene propylene polymer catheter (Jelco; Smiths Medical, Minneapolis, MN, USA) was used to puncture the skin at an angle of $30^{\circ}$ to $45^{\circ}$. After arterial blood was seen in the hub of the catheter, the catheter was dropped to $5^{\circ}$ to $10^{\circ}$ and slightly advanced to ensure cannulation of the artery. Finally, the needle was held in place while the catheter was advanced into the radial artery. In the GW group, after arterial blood was seen in the hub of catheter, the arterial catheter was advanced through the posterior wall of the radial artery. The needle was removed and the catheter withdrawn until arterial blood pulsation was seen. Half of the guidewire $\left(0.018^{\prime \prime} \times 93 / 4^{\prime \prime}\right)$ (Arrow; Teleflex, Wayne, PA, USA) was inserted, and the arterial cannula was then advanced into the radial artery.

Intraoperatively, all patients were maintained under general anaesthesia with an endotracheal tube. After induction of anaesthesia, radial artery cannulation was performed. All residents performed their radial artery cannulation technique without any suggestions from the attending anesthesiologists. When the residents had completed their procedure, the catheter was connected to the pressure transducer to obtain the arterial waveform.

Successful cannulation of the radial artery was defined as the appearance of an arterial waveform on the monitor after connection to the transducer. The insertion time was measured from the first needle puncture of the patient's skin to placement of the catheter into the radial artery. The number of attempts was counted as the number of needle advances through a skin puncture until the patient's blood was seen in the hub. Failure of cannulation was defined as no achievement of radial artery cannulation within two attempts or a procedural time of more than 15 minutes. In cases of failure, the arterial cannulation was performed by the attending anesthesiologist. The number of attempts and the cannulation time were recorded by nurse anesthetists who were not involved in this study. The record of procedural time was stopped when the arterial waveform was seen on the monitor or failure of cannulation. Other collected data were the patients' characteristics, ASA physical status, underlying diseases, vital signs before cannulation, and local haematoma formation after cannulation.

The sample size was calculated by the success rate of the direct puncture and guidewire-assisted techniques in a previous study[9] with a type I error of 0.05 and power of 0.8 . The result indicated that 29 
patients were required in each group.

Statistical analysis was performed using SPSS version 20.0 software (IBM Corp., Armonk, NY, USA). Continuous data are presented as mean \pm standard deviation or median (interquartile range). Categorical data are presented as number (percentage). The chi-square test or Fisher's exact test was performed to compare categorical variables, and Student's t-test or the Mann-Whitney $\mathrm{U}$ test was performed to compare continuous variables in each group. Kaplan-Meier curves of the cumulative success of cannulation and the procedural performance time were plotted. The log-rank test was performed for statistical comparison of the two groups. A P-value of $<0.05$ was considered statistically significant.

\section{Results}

The DP group comprised 15 first-year anesthesiology residency trainees, and the GW group comprised 30 surgical residency trainees.

A total of 60 patients participated in this study (30 in the GW group and 30 in the DP group). Table 1 shows the patients' demographic data in the two groups. There were no significant differences in age, body mass index, or comorbidities. Immediately before catheterisation, there were no significant differences in the baseline haemodynamic parameters between the two groups. 
Table 1

Patient characteristics

\begin{tabular}{|c|c|c|c|}
\hline & $\begin{array}{l}\text { Guidewire-assisted group }(n= \\
\text { 30) }\end{array}$ & $\begin{array}{l}\text { Direct arterial } \\
\text { puncture } \\
\text { group }(n=30)\end{array}$ & $\begin{array}{l}\mathrm{P} \\
\text { value }\end{array}$ \\
\hline Age, years & $57.3 \pm 14.2$ & $58.7 \pm 13.2$ & 0.693 \\
\hline $\mathrm{BMI}, \mathrm{kg} / \mathrm{m}^{2}$ & $22.4 \pm 3.2$ & $23.3 \pm 4.4$ & 0.401 \\
\hline Female sex & $11(36.7)$ & $17(56.7)$ & 0.121 \\
\hline ASA physical status & & & 0.093 \\
\hline 1 & $3(10.0)$ & $3(10.0)$ & \\
\hline 2 & $18(60.0)$ & $10(33.3)$ & \\
\hline 3 & $9(30.0)$ & $17(56.7)$ & \\
\hline \multicolumn{4}{|l|}{ Comorbidities } \\
\hline Diabetes mellitus & $7(23.3)$ & $6(20.0)$ & 0.754 \\
\hline Hypertension & $16(53.3)$ & $16(53.3)$ & 1.000 \\
\hline Coronary artery disease & $1(3.3)$ & $2(6.7)$ & 0.557 \\
\hline End-stage renal disease & $0(0.0)$ & $26.7)$ & 0.150 \\
\hline \multicolumn{4}{|c|}{ Before arterial catheterisation } \\
\hline $\begin{array}{l}\text { Systolic blood pressure, } \\
\text { mmHg }\end{array}$ & $116.7 \pm 18.1$ & $123.3 \pm 24.5$ & 0.262 \\
\hline $\begin{array}{l}\text { Diastolic blood pressure, } \\
\mathrm{mmHg}\end{array}$ & $66.8 \pm 10.6$ & $70.9 \pm 10.9$ & 0.148 \\
\hline Pulse rate, beats/min & $74.9 \pm 16.7$ & $79.4 \pm 13.0$ & 0.243 \\
\hline
\end{tabular}

The overall success rate of radial artery cannulation was 27 of $30(90.0 \%)$ patients in the GW group and 15 of $30(50.0 \%)$ patients in the DP group $(P=0.001)$. The first-attempt cannulation success rate was significantly higher in the GW than DP group (Table 2). 
Table 2

Success rate and cannulation time

\begin{tabular}{|llll|}
\hline & $\begin{array}{l}\text { Guidewire-assisted } \\
\text { group }(\mathbf{n}=\mathbf{3 0})\end{array}$ & $\begin{array}{l}\text { Direct arterial puncture group } \\
(\mathbf{n = 3 0})\end{array}$ & P value \\
\hline Overall success rate & $27(90.0)$ & $15(50.0)$ & 0.001 \\
\hline Number of attempts & $20(66.7)$ & $8(26.7)$ & 0.002 \\
First-attempt success rate & & & \\
\hline Second-attempt success rate & $7(23.3)$ & $7(23.3)$ & 0.892 \\
\hline Time to successful cannulation & $2.36 \pm 1.36$ & $2.46 \pm 1.44$ & 0.033 \\
\hline Procedural performance time, min & $2.24(1.39-3.28)$ & $4.47(2.06-8.05)$ & \\
\hline Data are presented as $\mathrm{n}(\%)$, mean \pm standard deviation, or median (interquartile range). & \\
\hline
\end{tabular}

The Kaplan-Meier curve of successful insertion of the arterial line by study group is shown in Fig. 1. The log-rank test showed a statistically significant difference between the two groups $(P=0.002)$. The mean successful cannulation time was not significantly different between the two groups. However, the total procedural performance time was significantly shorter in the GW than DP group. Most of the successful cannulation times in both groups were less than 5 minutes. Among successful cannulations, 24 (88.9\%) participants in the GW group spent less than 5 minutes to achieve successful radial artery cannulation, whereas 15 (100\%) participants in the DP group spent less than 5 minutes to achieve successful cannulation.

In terms of complications, a haematoma was found in 7 of $30(23.3 \%)$ patients in the GW group and in 11 of 30 (36.6\%) patients in the DP group. There was no significant difference between the two groups.

\section{Discussion}

In this study, the guidewire-assisted radial artery cannulation technique increased the overall success rate among inexperienced trainees from 50-90\%. Additionally, the first-attempt cannulation success rate was significantly higher with the guidewire-assisted technique than with direct arterial puncture.

The process of cannulation requires delicate and fine-tuned hand movements. Previous studies have shown low cannulation success rates in trainees with minimal or no experience ranging from 34.0$38.6 \%[5,10]$ Theoretically, learning how to perform radial artery cannulation is a psychomotor skill that requires a certain amount of practice and supervisor feedback to achieve success and gain experience. Thus, previous studies have shown that radial artery cannulation with the direct puncture technique performed by experienced clinicians has higher success rates ranging from 56.4-82.0\% depending on various factors such as the vessel size, catheter type, and performer's experience.[11, 12] During a training program, every trainee must practice to gain more experience with radial artery cannulation; however, this 
practice may involve either multiple cannulation attempts or failure, leading to procedure-related complications such as haematomas, vasospasm, infection, or vessel thrombosis.[13]

For inexperienced trainees, the determining factors of successful radial artery cannulation are identification of the appropriate puncture site of the radial artery and railroading the catheter into the artery. Therefore, new techniques and various equipment and tools have been developed to facilitate easier and faster radial artery cannulation. Beginners might not be confident with the artery palpation technique and may thus encounter difficulties in identification of the radial artery puncture site. This may result in misalignment of the puncture point over the radial artery. Moreover, the radial artery has a very small diameter at the level of the wrist (average diameter of 2.25-2.32 mm).[14, 15] The outer diameter of a 20-G catheter, which is the standard size for radial artery cannulation in adults, is $1.1 \mathrm{~mm}$. Consequently, this procedure is often difficult for inexperienced trainees. Ultrasonography is very useful for visualisation of the vessel underneath the body surface for visual projection instead of palpation. Previous studies have used ultrasound guidance to locate the position of the radial artery. However, Zhefeng et al.[16] found no benefit of ultrasound-guided puncture. They showed that the use of traditional real-time ultrasonic localisation of the artery by inexperienced trainees produced a cannulation success rate of only 34.2\%.[16] Another study involving senior anesthesiology residents who performed ultrasound-guided versus landmark-palpation radial artery cannulation showed no significant difference in the success rate between the two techniques (78\% vs. 82\%,respectively).[12] This authors stated that the lack of a significant difference could be explained by the learning curve of ultrasound-guided cannulation, which was a state-of-the-art and unfamiliar technique. In contrast, a recent study showed that ultrasound guidance increased the first-attempt success rate but not the overall cannulation success rate when compared with digital palpation.[3] As mentioned above, the ultrasound-guided technique facilitates precise visualisation of the artery location. However, the success rate of radial artery cannulation with ultrasound guidance was low and unsatisfactory. The present study involved participants who were inexperienced in radial artery cannulation and had been trained only by watching media or practicing on manikins. Nevertheless, this study showed that the effectiveness of watching short video media which demonstrated the steps of procedure in group of surgical residents would be sufficient for the performers. The success rate was higher in the guidewire-assisted group, which may be explained by the last step of radial artery cannulation (i.e., advancement of the catheter into the vessel). Beards et al.[9] noted that failure to cannulate the artery using the direct puncture technique commonly resulted from an inability to successfully advance the catheter despite the fact that the needle appeared to be within the artery. Additionally, studies involving paediatric patients showed that advancing the catheter after arterial wall penetration was more difficult than localisation of the artery.[17, 18] Mangar et al.[6] suggested that the causes of failure were tangential orientation of the catheter in relation to the artery, tortuosity of the vessel, and arterial vasospasm. In such cases, a guidewire can serve as a stent to facilitate advancement of the catheter through the skin and subcutaneous tissue and into the blood vessel.

In the present study, the overall procedural performance time was shorter in the guidewire-assisted group. Our study protocol allowed the participant trainees to perform two radial artery cannulation procedures 
within 15 minutes; however, most of the successful cannulations (either guidewire-assisted or by the direct puncture technique) occurred within the first 5 minutes. Because our facility is a teaching hospital, we balanced the need for the trainees to practice cannulation skills and the risk of injury to patients or a long procedure time. Therefore, the practice time for radial artery cannulation by inexperienced trainees was expected to be 5 to 10 minutes. Even in a study comparing ultrasound-guided radial artery cannulation, which is a newer technique, versus the direct puncture technique at different levels of residency training, most of the successful cannulations required less than 5 minutes. [5]

Our study has several limitations. Inevitably, there was some effect of first-time experience on arterial cannulation to the second performance in anesthesiology residents. This study could not know or assess the individual operators' learning experience from the prior cannulation. Secondly, our study did not evaluate the comprehensive assessment after our manikin- based training and watching video media in anesthesiology residents. The lack of understanding of the task affects on acquisition of procedural skills.[19] Lastly, we did not have a simulator training of radial artery cannulation in surgical residents. The success rate of cannulation might be altered in performers who have an effective procedural skill training.

\section{Conclusion}

Guidewire-assisted radial artery cannulation showed a significantly higher success rate and shorter procedural performance time. Guidewire-assisted radial artery cannulation has advantages over direct arterial puncture when performed by inexperienced trainees.

\section{Abbreviations}

ASA: American Society of Anesthesiologists

BMl: Body mass index

IQR: Interquartile range

mmHg: Millimetre of mercury

mm: Millimetre

SD: Standard deviation

\section{Declarations}

\section{Ethics approval and consent to participate}

The study was approved by ethics committee of Ramathibodi Hospital, Mahidol University, Bangkok, Thailand (ID 046125). Informed consent was obtained from all participants in the trial. The trial was 
retrospectively registered on Thai Clinical Trials Registry (TCTR20200601004). Date of registration was January 19,2020 . A late registration did not result in any changes in the study protocol. http://www.thaiclinicaltrials.org/page_user/\#

\section{Consent for publication}

Not applicable

\section{Availability of data and materials}

All the data used and/or analyzed are available from the corresponding authors upon reasonable request.

\section{Competing interests}

The authors declare that they have no competing interests.

\section{Funding}

The authors have no sources of funding to declare for this manuscript.

\section{Contributions}

WA processed data clearance, data analysis, data interpretation and drafted the manuscript, NB participated in designing and coordinating the study, IS contributed to data collection, prepared figures and tables, PL contributed to the study design and revised the manuscript. All author read and approved the final manuscript.

\section{Acknowledgements}

Assist.Prof. Surirat Srisawasdi donated arterial guidewires to our department for residency training program.

\section{Authors' information}

\section{Affiliations}

Department of Anesthesiology, Faculty of Medicine, Ramathibodi Hospital, Mahidol University, Thailand Worapot Apinyachon, Natthaporn Boonyakad, Inthuon Sangasilpa, Phisut Lavanrattanakul

\section{Corresponding author}

Correspondence to Phisut Lavanrattanakul

\section{References}


1. Esper SA, Pinsky MR. Arterial waveform analysis. Best Pract Res Clin Anaesthesiol 2014,28:363-80.

2. Brzezinski M, Luisetti T, London MJ. Radial artery cannulation: a comprehensive review of recent anatomic and physiologic investigations. Anesth Analg 2009,109:1763-81.

3. Bhattacharjee S, Maitra S, Baidya DK. Comparison between ultrasound guided technique and digital palpation technique for radial artery cannulation in adult patients: an updated meta-analysis of randomized controlled trials. Journal of clinical anesthesia 2018,47:54-9.

4. Nakayama Y, Inagaki Y, Nakajima Y, Sessler DI, Mukai N, Ogawa S, et al. A practical training program for peripheral radial artery catheterization in adult patients: a prospective, randomized controlled trial. Anesthesiology 2016,125:716-23.

5. Kiberenge RK, Ueda K, Rosauer B. Ultrasound-guided dynamic needle tip positioning technique versus palpation technique for radial arterial cannulation in adult surgical patients: a randomized controlled trial. Anesthesia and analgesia 2018,126:120-6.

6. Mangar D, Thrush DN, Connell GR, Downs JB. Direct or modified Seldinger guide wire-directed technique for arterial catheter insertion. Anesthesia and analgesia 1993,76:714-7.

7. Yildirim V, Ozal E, Cosar A, Bolcal C, Acikel CH, Kiliç S, et al. Direct versus guidewire-assisted pediatric radial artery cannulation technique. Journal of cardiothoracic and vascular anesthesia 2006,20:4850 .

8. Gronbeck C 3rd, Miller EL. Nonphysician placement of arterial catheters. Experience with 500 insertions. Chest 1993,104:1716-7.

9. Beards SC, Doedens L, Jackson A, Lipman J. A comparison of arterial lines and insertion techniques in critically ill patients. Anaesthesia 1994,49:968-73.

10. Li YH, Wang YQ, Zeng L, Chen W, Yang LY, Zhu QF, et al. Use of 1-ml hollow tube-assisted radial artery catheterization in clinical anesthesiology. International journal of clinical and experimental medicine 2014,7:2740-3.

11. Peters C, Schwarz SK, Yarnold CH, Kojic K, Kojic S, Head SJ. Ultrasound guidance versus direct palpation for radial artery catheterization by expert operators: a randomized trial among Canadian cardiac anesthesiologists. Canadian journal of anaesthesia $=$ Journal canadien d'anesthesie 2015,62:1161-8.

12. Tangwiwat $S$, Pankla W, Rushatamukayanunt $P$, Waitayawinyu $P$, Soontrakom $T$, Jirakulsawat $A$. Comparing the success rate of radial artery cannulation under ultrasound guidance and palpation technique in adults. J Med Assoc Thai 2016,99:505-10.

13. Scheer B, Perel A, Pfeiffer UJ. Clinical review: complications and risk factors of peripheral arterial catheters used for haemodynamic monitoring in anaesthesia and intensive care medicine. Crit Care 2002,6:199-204.

14. Beniwal S, Bhargava K, Kausik SK. Size of distal radial and distal ulnar arteries in adults of southern Rajasthan and their implications for percutaneous coronary interventions. Indian Heart J 2014,66:506-9. 
15. Ashraf T, Panhwar Z, Habib S, Memon MA, Shamsi F, Arif J. Size of radial and ulnar artery in local population. JPMA The Journal of the Pakistan Medical Association 2010,60:817-9.

16. Zhefeng Q, Luo C, Zhang L, Li X, He H, Chi P. Application of optimized ultrasonic localization system for radial artery puncture by intern doctors: a randomized trial. Med Sci Monit 2019,25:1566-71.

17. Cuper NJ, de Graaff JC, Hartman BJ, Verdaasdonk RM, Kalkman CJ. Difficult arterial cannulation in children: is a near-infrared vascular imaging system the answer? British journal of anaesthesia 2012,109:420-6.

18. Jang YE, Kim EH, Lee JH, Kim HS, Kim JT. Guidewire-assisted vs. direct radial arterial cannulation in neonates and infants: a randomised controlled trial. European journal of anesthesiology 2019,36:738-44.

19. Bould MD, Crabtree NA, Naik VN. Assessment of procedural skills in anaesthesia. Br J Anaesth. 2009,103:472-83.

\section{Figures}




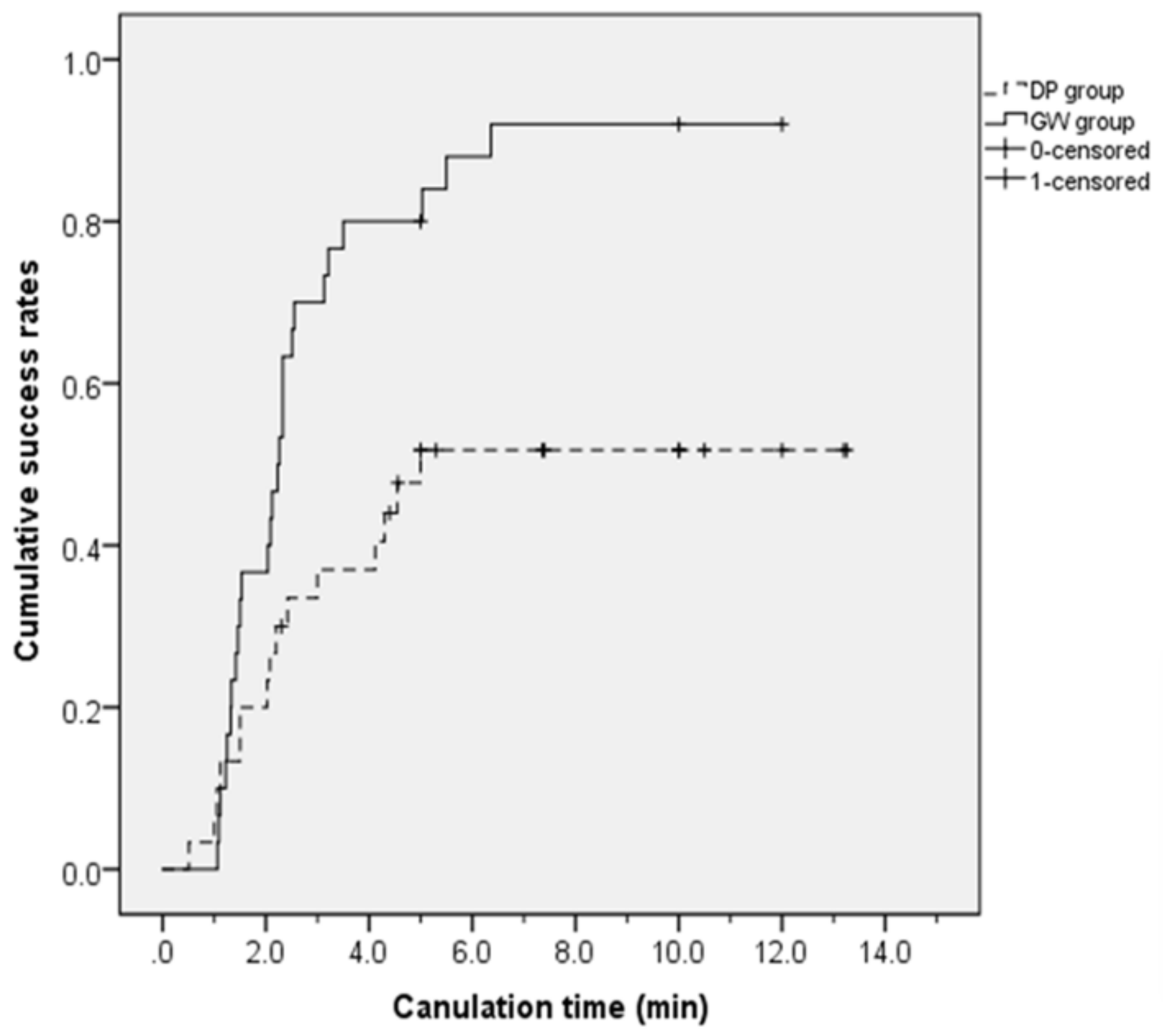

Figure 1

Kaplan-Meier curve of cumulative success rate. DP, direct arterial puncture, GW, guidewire-assisted cannulation 